\title{
The Fifth Force, the Sixth Force and the Spring Force
}

\author{
Ling Man Tsang ${ }^{1}$ \\ ${ }^{1}$ Fisica Laboratorio, TIS, Block K, Macau University of Science and Technology, Taipa, Macau \\ Correspondence: Ling Man Tsang, Fisica Laboratorio, TIS, Block K, Macau University of Science and \\ Technology, Taipa, Macau. E-mail: lmtsang05@hotmail.com
}

Received: January 13, 2012

Accepted: February 11, 2012 Online Published: May 1, 2012

doi:10.5539/apr.v4n2p229

URL: http://dx.doi.org/10.5539/apr.v4n2p229

\begin{abstract}
Upon revisiting the famous Pound-Rebka-Snider experiments on the gravitational red-shift, the fifth and even the sixth force in nature are in fact the extension and compression of the spring, where the spring term is the cosmological constant in Einstein's field equations.
\end{abstract}

Keywords: cosmological constant

\section{Introduction}

In spite of the success of special relativity, scientists still believe that the entire space is non-vacuumic but filled up with materials such as the Higg's particles, dark matters and etc. The Aharonov-Bohm experiment indicates the vacuum is physical. Since light travels through the physical vacuum, we can call the medium aether. These aether are elastic materials that can either be extended or compressed. They attach parasitically onto the sources. Our earth carries these elastic matters or known as springs while revolving around the sun, causing the null result of the Michelson-Morley experiments.

\section{The Spring Theory}

A falling object is compressing the spring while an escaping object is stretching it. Here are the two equations of an object at a certain height above the earth's surface:

$$
\begin{gathered}
\frac{G M}{r_{o}^{2}}-\omega^{2} r_{0} \cos ^{2} \theta-k r_{0}=\text { acceleration of a falling body } \\
\frac{G M}{r_{o}^{2}}-\omega^{2} r_{0} \cos ^{2} \theta+k r_{0}=\text { deceleration of an escaping body }
\end{gathered}
$$

The first term is the usual Newton's inverse square law, the second term, or the centrifugal acceleration, depends on the latitude of the body. For $\theta=0$, the centrifugal acceleration at the equator will be maximum. The spring constant $k$, which can either be extended or compressed, is in fact the cosmological constant if we look at Einstein's exterior field equations of the form

$$
R_{\mu v}=\Lambda g_{\mu v}
$$

where $k=\frac{2 \Lambda}{3}$

The spring term $k r$ is so-called since it resembles an harmonic oscillator.

Taking the earth rotation

the earth radius

$$
\omega=7.3 \times 10^{-5} / \mathrm{sec}
$$

$$
r_{0}=6.4 \times 10^{6} \mathrm{~m},
$$

the earth mass $=6 \times 10^{24} \mathrm{~kg}, \theta=42^{\circ}$, the latitude of Massachusetts where Pound and Rebka performed their experiments at Harvard.

The above two equations become 


$$
\begin{gathered}
9.7516-k r=\text { acceleration } \\
9.7516+k r=\text { deceleration }
\end{gathered}
$$

\section{Re-analysis of the Pound-Rebka-Snider Experiments}

The Jefferson Physical Laboratory at Harvard used a ${ }^{57} \mathrm{Fe}$ source being placed at a height of $22.6 \mathrm{~m}$ above the detector. Gamma photons dropped to the detector. The original purpose of this Pound-Rebka experiment was to demonstrate the effect on photons under the earth's gravity. The following data can be found in many textbooks(see Gravitation by Misner/Thorne/Wheeler):

$\Delta r=$ height dropped $22.6 \mathrm{~m}$

$$
h \Delta v=h\left(v-v_{\mathrm{o}}\right)=3.5 \times 10^{-11} \mathrm{eV}
$$

$E=h v_{\mathrm{o}}$ the source energy $14.4 \mathrm{keV}$

$h=$ Planck's constant

We obtain

$$
a=\frac{c^{2} \Delta E}{E \Delta r}=9679 \mathrm{~m} / \mathrm{s}^{2},
$$

which is only true at Harvard, or likewise the State of Massachusetts.

In 1965 Pound and Snider improved the apparatus so that the energy shifts on the upward and downward path gave a measured difference of

$$
\left(\frac{\Delta E}{E}\right)_{\text {down }}-\left(\frac{\Delta E}{E}\right)_{u p}=4.905 \times 10^{-15}
$$

Since the first term of (4) is known, the second term will immediately yield the deceleration of $9.854 \mathrm{~m} / \mathrm{s}^{2}$.The acceleration and deceleration become

$$
\begin{aligned}
& 9.7516-k r=9.679 m / s^{2} \\
& 9.7516+k r=9.854 m / s^{2}
\end{aligned}
$$

This is more than a coincidence to have $9.679 \mathrm{~m} / \mathrm{s}^{2}$ smaller than $9.7516 \mathrm{~m} / \mathrm{s}^{2}$ in $(1 \mathrm{~b})$ together with 9.854 $\mathrm{m} / \mathrm{s}^{2}$ larger than $9.7516 \mathrm{~m} / \mathrm{s}^{2}$ in $(2 \mathrm{~b})$. The existence of an extra term in both equatioins is necessary. The earth's spring from both ( $1 \mathrm{~b})$ and $(2 \mathrm{~b})$ is found to be

$$
k=1.2198 \times 10^{-8} / \mathrm{s}^{-2}
$$

Obviously, (1b) and (2b) violate the Principle of Equivalence, that is,

gravitational mass $\mathrm{x}$ gravitational field strength $\neq$ inertial mass $\mathrm{x}$ acceleration. Later in this paper, we will show that the sixth force can overcome the gravitational pull, causing the universe to expand.

A striking prediction is, at a far distance $R=32,000 \mathrm{~km}$ from earth, the spring force balances Newton's inverse square law. To express mathematically;

$$
\begin{array}{ccc}
G M / r^{2}-k r=a & \text { where } & r<32,000 \mathrm{~km} \\
G M / r^{2}-k r=0 & \text { where } & r=R=32,000 \mathrm{~km} \\
G M / r^{2}=a & \text { where } & r>32,000 \mathrm{~km}
\end{array}
$$

The fifth force against earth's gravity is of intermediate range while the one (2b) having the same direction with gravity is so-called the sixth force (New Scientist, 1988). These two fprces are simply the extension and compression of the earth's spring. We need to point out that the Yukawa-like potential for the fifth force (Sanders 1984, 1990; Fischbach 1986, 1992, see Tsang 2010, 2012) may yield a non-logical result such as at $a=0, r$ cannot be defined. In spite of the difficulty to search for this fifth force, theories do exist. 


\section{Search of the Fifth Force on the Moon}

Only the earth and the moon can provide solid grounds to verify (1) and (2). Regretfully, none of the NASA's and China's Chang E lunar projects ever focused on the moon's gravity (Astronomy, 2011; Ou Yang, 2011). In fact, a simple free fall experiment onto the moon's surface can verify the existence of the fifth force. The time taken for a free falling body dropping from a certain height onto the moon's surface is found to be (Tsang, 2012).

$$
T>\sqrt{\frac{R+H}{2 G M}}\left(-\sqrt{r((H+R-r}+(H+R) \arcsin \sqrt{\frac{r}{R+H}}\right)_{R+H}^{R}
$$

Taking the height of drop $\mathrm{H}=59,000 \mathrm{~m}, \mathrm{~T}>259 \mathrm{~s}$. To check this, we use the simple equation of $H=\frac{1}{2} g t^{2}$, where $a=1.627 \mathrm{~m} / \mathrm{s}^{2}$, and obtain $\mathrm{t}=270 \mathrm{~s}$.

Thus, (6) is reasonable. Eventually, the spring of the moon can be obtained as

$$
k=\frac{1}{T^{2}}\left(\frac{\pi}{2}-\arcsin \sqrt{\frac{R}{R+H}}\right)^{2}
$$

The moon's almost vacuumic surface provides a good conditionto verify (6) as well as the fifth force. The sixth force is in fact the opposite sign of the fifth force. Therefore, each source should have its own spring constant as predicted (Tsang, 2010, 2012).

\section{The Existence of Spring or Aether}

The aether drift experiments can be traced back as early as 1925 from the two papers of Miller (Miller, 1925). Various papers did improve the Michelson-Morley experiments to verify the aether drift (Atsukovsky, 1990, Galaey, 2002) by considering that aether is fluid. Galaey even found out the kinematic viscosity of aether to be $6.24 \times 10^{-5} \mathrm{~m}^{2} \mathrm{sec}^{-1}$. Marmet (Marmet, 2000) had discussed the case of light travel between San Francisco and New York both of which are at the same latitude. The result was that light travelling from San Francisco to New York takes $28 \mathrm{~ns}$ longer than light travelling westward. There are still various research papers on aether (Shtyrkov, 2005; Gift, 2012; and references therein). Finally, we deduce that aether or spring sea is compressible viscous fluid. Further conclusion is that the cosmological constant refers to a 3 dimensional spring type of object which is unseen and can be treated as dark matters filling up the entire space. The cosmological term must be larger than the gravitational pull of the universe in the form of $(k=\Lambda)$

$$
\Lambda r-G M / r^{2}=\text { acceleration }
$$

thus causing the expansion. This can only happen once the spring becomes a sixth force. The above (7) can be re-written as

$$
(\Lambda-G \rho) r=a=v d v / d r
$$

or $\mathrm{G} \rho<\Lambda$

where

$$
\begin{gathered}
M=\frac{4}{3} \pi r^{3} \rho=\text { total mass of our universe } \\
\mathrm{G} \sim 10^{-11} \mathrm{~m}^{3} / \mathrm{sec}^{2} / \mathrm{kg} \\
\rho \sim 10^{-27} \mathrm{~kg} / \mathrm{m}^{3}=\text { mass density inside the universe }
\end{gathered}
$$

From (8), our universe is accelerating.and immediately we obtain the approximate relation of $H \sim \Lambda^{1 / 2}$, where $\mathrm{H}$ $=$ Hubble constant $\left(10^{-18} \sim 10^{-17.5} / \mathrm{s}\right)$. As we can see that the Hubble constant is also 3 dimensional, which is the square root of the spring constant of our universe.

\section{Conclusion}

Besides large scale structures, spring theory can also be applied to the micro-world. Nuclear matters such as neutron-proton are connected by springs. This approach can avoid the complexity of multi-dimensional string theory. Our physical world can be explained by 3 dimensional physics. In case aether exists, the velocity of light will not be a constant. The foundations of relativity may collapse. 


\section{References}

Astronomy. (2011). The moon's surface mapped in detail, Jan. p.24 Secrets beneath the moon's surface, March p.24.

Atsukovsky, V. A. (1990). General ether dynamics. Simulation of the matter structures and fields on the basis of the ideas about the gas-like ether. Energoatomizdat, Mpscow 280. (in Russian)

Fischbach, E, Sudarsky, D, Szafer, A, Talmadge, C. \& Aronson, S. H. (1986). Reanalysis of the Eötvös experiment. Phys. Rev. Lett., 56, 3-6. http://dx.doi.org/10.1103/PhysRevLett.56.3

Fischbach, E., \& Talmadge, C. (1992). Six years of the fifth force. Nature, 356, 207-215. http://dx.doi.org/10.1038/356207a0

Galaey, Y. M. (2002). The measuring of ether drift velocity and kinematic ether viscosity within optical waves band. Spacetime and Substance, 3, 5(15), 207-224.

Gift, S. J. (2010). Successful search for ether drift in a modified Michelson-Morley experiiment using the GPS, Applied Physics Research, 4(1), 185-192.

Marmet, P. (2000). The GPS and the constant velocity of light. Acta Scientiarum, 22, 1269-1279.

Miller, D. C. (1925). Ether drift experiments at Mount Wilson. Proc. Nat. Acad. Amer. 11, 306-314.

Miller, D. C. (1925). Significance of the ether drift experiments of 1925 at Mount Wilson. Science, 68, 433-443. http://dx.doi.org/10.1073/pnas.11.6.306

New Scientist. (1988). 17 $7^{\text {th }}$ December. In fact,there is no technical article on this topic up to present. See also Will, C. M. (1993). Was Einstein Right? Basic Books, $2^{\text {nd }}$ edition.

OuYang, Ziyuan. (2010). J. Macau Univ. Sc. \& Technol., 4(1), 12-24.

Sanders, R. H. (1984). Anti-gravity and galaxy rotation curves. Astron. Astrophys., 136, 21-23.

Shtyrkov, E. I. (2005). Observation of ether drift in esperiments with Geostationary satellites, Ptoceedingd of the National Philosophy Alliance. $12^{\text {th }}$ Annual Conference,Storrs CT, 23-27, May, 201-205.

Tsang, L. M. (2010). Some Static Spherical Classical Solutions including the Cosmological term.Can. J. Pure Appl. Sc., 4(1), 1073-1079.

Tsang, L. M. (2012). How can NASA's Lunar Reconnaisance Orbiter projects verify the existence of the Fifth Force. New Astronomy, 17, 18-21. http://dx.doi.org/10.1016/j.newast.2011.05.004 